
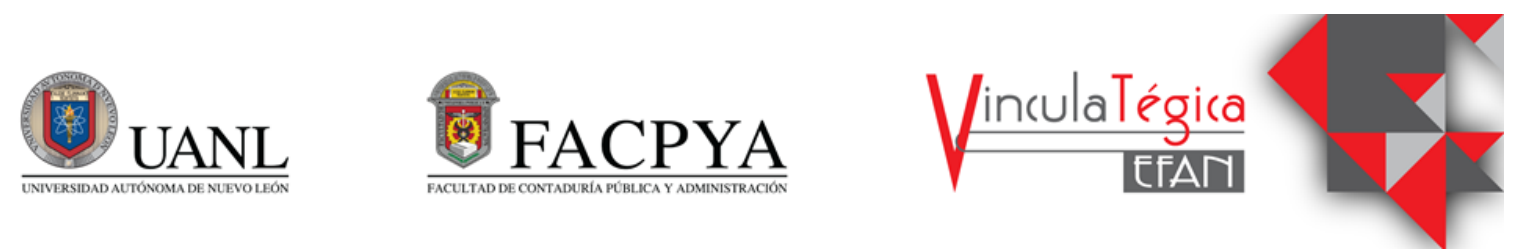

\title{
Impacto de la crisis hipotecaria financiera del 2008 en la estructura de capital en las empresas públicas mexicanas
}

\author{
Ana Castro Spinelli ${ }^{1}$ y Michael Demmler ${ }^{2}$

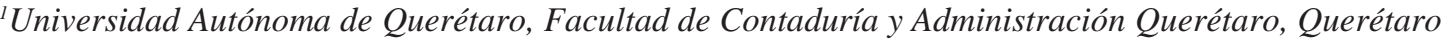 \\ México, castroac1@yahoo.com.mx, Cerro de las Campanas s/n, Cp. 76010, (+52) 4421921200 \\ (Times New Roman, 10, cursiva) \\ ${ }^{2}$ Universidad Autónoma de Querétaro, Facultad de Contaduría y Administración Querétaro, Querétaro \\ México, michael.demmler01@ gmail.com, Cerro de las Campanas s/n, Cp. 76010, (+52) 4421921200
}

\author{
Información del artículo revisado por pares \\ Fecha de aceptación: junio-2021 \\ Fecha de publicación en línea: diciembre-2021 \\ DOI: https://doi.org/10.29105/vtga7.1-168
}

\begin{abstract}
Resumen
El presente estudio tiene la finalidad de identificar el impacto de la crisis hipotecaria financiera del 2008 en la estructura de capital en las empresas públicas mexicanas listadas en el Índice de Precios y Cotizaciones (IPC) de la Bolsa Mexicana de Valores (BMV). Se realizó el análisis tanto para el total de las empresas como para grupos de empresas clasificadas de acuerdo con su retorno sobre los activos (ROA) y al valor de sus activos. Se encontró que el apalancamiento general de todos los grupos incrementó de 2007 a 2009, siendo que el primer año el apalancamiento por medio de pasivos circulantes incrementó una mayor proporción que el financiamiento a largo plazo; en el segundo año, el efecto a largo plazo se incrementó y el de corto plazo se redujo. Se sugieren abrir líneas de investigación para identificar las causas por las cuales el apalancamiento de las empresas se homogenizó a lo largo de los años, y si los resultados son coincidentes con el apalancamiento de la actual crisis económica derivada por la pandemia del SARS-COV-2.
\end{abstract}

Palabras clave: crisis hipotecaria, crisis del 2008, estructura de capital, decisiones financieras, financiamiento.

Códigos JEL: G32, G17, G12, G30

\section{INTRODUCCIÓN}

La crisis hipotecaria de 2008 causó estragos en todo el mundo, sobre todo en las economías que tienen una fuerte relación comercial con
Abstract

The aim of this study is to identify the impact of the 2008 financial mortgage crisis on the capital structure of Mexican public companies listed in the Price and Quotation Index (IPC) of the Mexican Stock Exchange (BMV). The analysis was carried out both for the total number of companies and for groups of companies classified according to their return on assets (ROA) and the value of their assets. It was found that the general leverage of all groups increased from 2007 to 2009, being that the first year the leverage through current liabilities increased a greater proportion than the long-term financing; in the second year, the long-term effect increased and the short-term effect decreased. It is suggested to open lines of investigation to identify the causes by which the leverage of the companies became homogenized over the years, and if the results coincide with the leverage of the current economic crisis derived from the SARS-COV-2.

Key words: subprime crisis, capital structure, financial decisions, financing, indebtedness.

JEL Codes: G32, G17, G12, G30

Estados Unidos como la que tiene con México. La deficiente gestión de créditos en Estados Unidos fue el origen de la crisis. En consecuencia, se desencadenó la quiebra de varios bancos en el país, siendo que la Reserva 
Federal abrió líneas de crédito, adquirió valores comerciales de cartera privada y redujo las tasas de intervención. Por su parte, las consecuencias para México fueron la baja de remesas por parte de los trabajadores migrantes y la contracción del comercio internacional (Rivera y Campuzano, 2009; Correa, 2010; Ocampo, 2009; Calderón y Bielma, 2017). Varios autores señalan que las crisis financieras traen como resultado cambios en el apalancamiento en las empresas, siendo que la deuda a largo plazo es sustituida por deuda a corto plazo (Alves y Francisco, 2015; Christensen et al., 2015; Lopes, 2017; Puntaier, 2010; González, 2014 y Custódio et al., 2013).

El presente estudio tiene la finalidad de identificar el impacto de la crisis hipotecaria financiera del 2008 en la estructura de capital en las empresas públicas mexicanas listadas en el Índice de Precios y Cotizaciones (IPC) de la Bolsa Mexicana de Valores (BMV). Para fines de este estudio la pregunta de investigación fue: ¿Cuál fue el impacto de la crisis financiera del 2008 en la estructura de capital en las empresas públicas mexicanas listadas en el IPC?

Para la realización de este estudio se tomaron empresas cuyos datos estaban en pesos mexicanos y que tenían datos de 20042016. Los datos se obtuvieron de la base de datos de Standard \& Poors (2021).

Los resultados de la investigación son coincidentes con Christensen et al. (2015), Lopes (2017), Alves y Francisco (2015), Puntaier (2010), González (2014) y Custódio et al. (2013); en tanto que el apalancamiento incrementó durante la crisis hipotecaria del 2008 para 15 empresas públicas mexicanas listadas en el Índice de Precios y Cotizaciones (IPC) de la Bolsa Mexicana de Valores (BMV). También es coincidente con los autores con respecto a que la deuda a largo plazo es sustituida por deuda a corto plazo en el año de la crisis; sin embargo, un año después la deuda a corto plazo se reduce e incrementa la deuda a largo plazo.

De esta forma, el trabajo se estructura de la siguiente manera: en el apartado dos, se presentará el marco teórico que describe tanto el impacto de la crisis financiera hipotecaria del 2009 en México y en la estructura de capital de las empresas en general, como la concepción de la estructura de capital y mediciones del apalancamiento. En la sección tres se establece la metodología para la realización de un estudio de carácter cuantitativo donde se estudian las variables de pasivos totales / activos totales, pasivos a largo plazo / activos totales y pasivos circulantes / activos totales. Así mismo, en esta sección se determinan los diferentes análisis realizados, empezando por estadística descriptiva; seguido por pruebas $\mathrm{t}$ para determinar si la comparación de medias por año de las variables mencionadas muestra que éstas son estadísticamente iguales; y se termina con análisis ANOVA donde se identifican las diferencias de la media del crecimiento de activos totales con respecto a pasivos totales, pasivos a largo plazo y pasivos circulantes para los años 2008 y 2009. En la sección cuatro se presentarán los resultados y la discusión de los mismos. Por último, en la sección cinco, se presentan las conclusiones a las cuales se llegaron en este estudio.

\section{MARCO TEÓRICO}

\subsection{Crisis hipotecaria financiera $y$ efecto en el apalancamiento.}

La crisis hipotecaria de 2008 originada en Estados Unidos, también llamada subprime, se extendió globalmente. Se pueden identificar dos factores importantes que desataron la crisis. Uno de ellos es la creciente oferta y demanda de bienes raíces. El segundo se refiere a la deficiente gestión de títulos financieros que se describe a continuación. En 2004 se registra la liberalización de los requisitos de capital de la banca de inversión, desregularización de hipotecas de baja calidad, propagación de derivados financieros llamados "permutas de riesgo de incumplimiento del deudor" (credit default swaps), incremento de activos financieros que no estaban incluidos en las hojas de balance de las instituciones financieras (sino que más bien permanecían en manos de intermediarios no regulados que fungían como fondos de inversión alternativos (hedge funds), y captación de recursos a corto plazo para 
invertirlos a largo plazo. Todo lo anterior llevó a incrementar el apalancamiento tanto personal como empresarial (Ocampo, 2009).

En consecuencia, el crédito se paralizó, las entidades financieras se descapitalizaron y se contrajo la actividad productiva de países industrializados. Estados Unidos sufrió la quiebra de algunos bancos como Bear Stearns que demeritó el mercado de valores que estaba respaldado por hipotecas, lo que ocasionó inmediata desconfianza en el mercado. Para solventar la situación, la Reserva Federal, en aras de mejorar la liquidez, empezó a abrir líneas de crédito al sector privado, a adquirir valores comerciales (de cartera privada) y a reducir las tasas de intervención reflejadas primeramente en los bonos del Tesoro a corto plazo, el mercado interbancario y las tasas tanto de depósito y crédito del sistema bancario; y después al mercado de capitales e hipotecario (Ocampo, 2009).

Ocampo (2009) menciona que los mecanismos de transmisión de la crisis hacia otros países como México fueron: la baja de remesas por parte de trabajadores migrantes y la contracción de comercio internacional. Calderón y Bielma (2017) puntualizan que los efectos para México fue una contracción de $6 \%$ de 2007 a 2009, teniendo una recuperación en 2010; adicionalmente, los autores indican que, este el ciclo económico duró 7.5 años.

Rivera y Campuzano (2009) indican que los principales efectos de la crisis se basan en la volatilidad en las tasas de interés, tipo de cambio, precios internacionales de hidrocarburos y de precios de productos y servicios. Así, por la fuerte relación en la balanza comercial entre México y Estados Unidos, México se vio afectado por la fuga de capitales ya que las empresas domésticas más importantes demandan fuertes fondos externos y tienen muchos pasivos en dólares. (Rivera y Campuzano, 2009; Correa, 2010).

Una de las principales afectaciones de la crisis hipotecaria financiera del 2008 fue el efecto en el apalancamiento. Alves y Francisco (2015) y Christensen et al. (2015) mencionan en momentos de crisis financieras las empresas suelen incrementar el apalancamiento general. Varios autores establecen que los inversionistas e intermediarios financieros se volvieron más aversos al riesgo, de forma tal que se incrementaron las tasas de interés y costos de agencia tanto de emisión de bonos como de contratación de deuda a largo plazo (Lopes, 2017; Alves y Francisco, 2015; Puntaier, 2010; González, 2014 y Custódio et al., 2013). Esto, ligado a un incremento de asimetrías de información, ocasionó la sustitución de deuda a largo plazo por la deuda a corto plazo (Lopes, 2017; Alves y Francisco, 2015; Puntaier, 2010; González, 2014 y Custódio et al., 2013).

Algunos autores como Lopes (2017), Myers $(1984 ; 2001)$ y Rivera y Campuzano (2009) aseguran que la estructura de capital en las empresas responde a restricciones y objetivos que las mismas empresas se establecen en cuanto a la relación deuda/activos totales. Así, se ha documentado que el refinanciamiento de deuda a largo plazo se puede obstaculizar ya que, debido a la sustitución de deuda de largo plazo por deuda de corto plazo, los flujos de efectivo se acortan o limitan (Jensen y Meckling, 1976; Jensen, 1986) y además aumenta el riesgo de impago, el riesgo de adquisición o contratación de financiamiento en un futuro y el riesgo de quiebra (Alves y Francisco, 2015; Jensen y Meckling, 1976; Jensen, 1986). Por lo tanto, la calificación crediticia de las empresas se ve afectada y las tasas de interés suben (Alves y Francisco, 2015). Custódio et al. (2013) indican que la deuda a corto plazo debe ser renegociada con mayor frecuencia, lo que ocasiona que las condiciones de la financiación se vean afectadas por una crisis financiera.

Chen et al. (2018), Zeitun et al. (2017) y Lopes (2017) aseguran que el otorgamiento de financiamiento por medio de deuda a largo plazo (en forma bonos o préstamos bancarios) depende de las características de la empresa. Uno de los aspectos a tomar en cuenta son la posesión de activos tangibles (o fijos) ya que sirven como garantía (Chen et al., 2018; Lopes, 2017). Otro factor decisivo es la perspectiva de crecimiento de la empresa (Zeitun et al., 2017).

\subsection{Estructura de capital y}




\section{apalancamiento.}

Existe coincidencia en cuanto a la definición de estructura de capital entre diversos autores (proporción de pasivos y capital con respecto a los activos de una empresa o la distribución del financiamiento); sin embargo, no hay un consenso con respecto a la medición de la estructura de capital. Algunos autores mencionan que las razones financieras que miden la estructura de capital son las razones de apalancamiento que se refieren a la razón de deuda total a activos y la razón de deuda a capital (Myers, 1984, 2001; Modigliani y Miller, 1958; 1963; Miller 1977; Stiglitz, 1969; Fama y French, 2002; Shyam-Sunder y Myers, 1999; Donaldson, 1961; Arias et al., 2009; Rodríguez, 2003).

Modigliani y Miller (1958) establecen que las empresas buscan la maximización de utilidades, a través de la búsqueda de proyectos cuyos rendimientos sean superiores al costo de capital (expresado en su Proposición III). Así, la inversión de proyectos incrementará la base de activos, seguido por la necesidad de financiamiento. Por esto, los ejecutivos de una empresa deben tomar la decisión de financiarse tanto de deuda como de capital (Jensen y Meckling, 1976). En consecuente, la elección de financiamiento puede cambiar la rentabilidad de la empresa (Modigliani y Miller, 1963; Miller 1977; Jensen y Meckling, 1976; Jensen, 1986) y por lo tanto afectar el retorno sobre los activos.

\section{MÉTODO}

El objetivo de este estudio es identificar el impacto de la crisis financiera del 2008 en la estructura de capital en las empresas públicas mexicanas listadas en el Índice de Precios y Cotizaciones (IPC) de la Bolsa Mexicana de Valores (BMV). Para la realización de este estudio se utilizó la base de datos de Standard \& Poors ${ }^{\circledR}$ de donde se obtuvieron datos anuales de empresas de 13 años que comprenden de los años fiscales 2004-2016. Este periodo de tiempo se eligió dado que, según Calderón y Bielma (2017), el ciclo de la crisis en México derivada de la crisis hipotecaria de Estados Unidos duró de 2007 a 2014. Además, es importante visualizar la tendencia de la estructura de capital antes y después de la crisis. Se obtuvo una muestra por conveniencia, conformada de 15 empresas públicas mexicanas pertenecientes al Índice de Precios y Cotizaciones (IPC) mostradas en el Apéndice I; éstas cotizaron en la Bolsa Mexicana de Valores (BMV) a lo largo de los 13 años de este estudio. Se omitieron las empresas clasificadas en el sector de "servicios financieros" según la BMV dado que tienen regulaciones específicas y alto apalancamiento por lo que pueden alterar los resultados (Skopljak y Luo, 2012). Por lo anterior, esta investigación se clasifica como cuantitativa longitudinal.

En la Tabla 1 se observan las variables que se tomarán en cuenta para el análisis estadístico de este estudio a las cuales se les denominará "variables del estudio" y serán: pasivos totales / activos totales, pasivos totales a largo plazo / activos totales y pasivos circulantes totales / activos totales (ver Tabla).

Tabla 1. Variables obtenidas de Standard \& Poors (2021) y variables calculadas.

\begin{tabular}{ccc}
\hline Nombre de la variable & Formato de la variable & Cálculo \\
\hline P/A & Porcentaje & 'Pasivos totales' / 'Activos totales' \\
PL/A & Porcentaje & 'Pasivos a largo plazo totales' / 'Activos totales' \\
PC/A & Porcentaje & 'Pasivos circulantes totales' / 'Activos totales' \\
\hline
\end{tabular}

Fuente: Elaboración propia.

Se realizaron dos clasificaciones con la finalidad de identificar si algún grupo de empresas mostraba algún cambio en cuanto a

la estructura de capital de las empresas (ver Tabla 2).

Tabla 2. Clasificación de las empresas según activos totales y ROA.

Activos $\quad$ Activos totales $\quad$ ROA




\begin{tabular}{|c|c|c|c|c|}
\hline & totales & & & \\
\hline Empresa & $\begin{array}{l}\text { Valor (en } \\
\text { millones } \\
\text { de pesos) }\end{array}$ & Clasificación & Valor & Clasificación \\
\hline Alfa, S. A. B. de C. V. & $\$ 110,970$ & $\begin{array}{c}\text { Mayores o iguales a } \$ 50,0000 \\
\text { millones de pesos }\end{array}$ & $3.03 \%$ & Menor de $6 \%$ \\
\hline Arca Continental, S.A.B. de C.V. & $\$ 21,659$ & $\begin{array}{c}\text { Menores de } \$ 50,000 \text { millones de } \\
\text { pesos }\end{array}$ & $10.08 \%$ & Mayor o igual a $6 \%$ \\
\hline CEMEX, S.A.B. de C.V. & $\$ 623,622$ & $\begin{array}{l}\text { Mayores o iguales a } \$ 50,0000 \\
\text { millones de pesos }\end{array}$ & $1.49 \%$ & Menor de $6 \%$ \\
\hline $\begin{array}{l}\text { El Puerto de Liverpool, S.A.B. de } \\
\text { C.V. }\end{array}$ & $\$ 55,504$ & $\begin{array}{c}\text { Mayores o iguales a } \$ 50,0000 \\
\text { millones de pesos }\end{array}$ & $7.63 \%$ & Mayor o igual a $6 \%$ \\
\hline $\begin{array}{l}\text { Fomento Económico Mexicano, } \\
\text { S.A.B. de C.V. }\end{array}$ & $\$ 187,345$ & $\begin{array}{l}\text { Mayores o iguales a } \$ 50,0000 \\
\text { millones de pesos }\end{array}$ & $5.79 \%$ & Menor de $6 \%$ \\
\hline Gruma, S.A.B. de C.V. & $\$ 44,435$ & $\begin{array}{c}\text { Menores de } \$ 50,000 \text { millones de } \\
\text { pesos }\end{array}$ & $3.17 \%$ & Menor de $6 \%$ \\
\hline $\begin{array}{l}\text { Grupo Aeroportuario del Centro } \\
\text { Norte, S.A.B. de C.V. }\end{array}$ & $\$ 9,860$ & $\begin{array}{c}\text { Menores de } \$ 50,000 \text { millones de } \\
\text { pesos }\end{array}$ & $6.88 \%$ & Mayor o igual a $6 \%$ \\
\hline $\begin{array}{c}\text { Grupo Aeroportuario del Pacífico, } \\
\text { S.A.B. de C.V. }\end{array}$ & $\$ 28,142$ & $\begin{array}{c}\text { Menores de } \$ 50,000 \text { millones de } \\
\text { pesos }\end{array}$ & $5.97 \%$ & Menor de $6 \%$ \\
\hline $\begin{array}{c}\text { Grupo Aeroportuario del Sureste, } \\
\text { S. A. B. de C. V. }\end{array}$ & $\$ 17,375$ & $\begin{array}{c}\text { Menores de } \$ 50,000 \text { millones de } \\
\text { pesos }\end{array}$ & $7.44 \%$ & Mayor o igual a $6 \%$ \\
\hline Grupo Bimbo, S.A.B. de C.V. & $\$ 58,506$ & $\begin{array}{l}\text { Mayores o iguales a } \$ 50,0000 \\
\text { millones de pesos }\end{array}$ & $5.08 \%$ & Menor de $6 \%$ \\
\hline Grupo Carso, S.A.B. de C.V. & $\$ 91,099$ & $\begin{array}{l}\text { Mayores o iguales a } \$ 50,0000 \\
\text { millones de pesos }\end{array}$ & $9.35 \%$ & Mayor o igual a $6 \%$ \\
\hline Grupo Televisa, S.A.B. & $\$ 122,852$ & $\begin{array}{c}\text { Mayores o iguales a } \$ 50,0000 \\
\text { millones de pesos }\end{array}$ & $5.45 \%$ & Menor de $6 \%$ \\
\hline Industrias Peñoles, S.A.B. de C.V. & $\$ 52,712$ & $\begin{array}{l}\text { Mayores o iguales a } \$ 50,0000 \\
\text { millones de pesos }\end{array}$ & $8.25 \%$ & Mayor o igual a $6 \%$ \\
\hline $\begin{array}{c}\text { Promotora y Operadora de } \\
\text { Infraestructura, S. A. B. de C. V. }\end{array}$ & $\$ 14,227$ & $\begin{array}{c}\text { Menores de } \$ 50,000 \text { millones de } \\
\text { pesos }\end{array}$ & $7.02 \%$ & Mayor o igual a $6 \%$ \\
\hline $\begin{array}{c}\text { Wal-Mart de México, S.A.B. de } \\
\text { C.V. }\end{array}$ & $\$ 118,464$ & $\begin{array}{c}\text { Mayores o iguales a } \$ 50,0000 \\
\text { millones de pesos }\end{array}$ & $11.35 \%$ & Mayor o igual a $6 \%$ \\
\hline
\end{tabular}

Fuente: Elaboración propia.

La primera clasificación tomó en cuenta el valor de los activos dado que las empresas buscan invertir en proyectos de inversión y dicha inversión debe ser financiada por mercado de deuda o de capital (Modigliani y Miller, 1958). Este grupo incluyó empresas con activos menores a $\$ 50,000$ millones de pesos, y otro grupo incluyó empresas con activos iguales o mayores $\$ 50,000$ millones de pesos (que se registraron en 2008). La segunda clasificación se realizó en función del retorno sobre los activos (ROA) dado que la decisión de financiamiento puede afectar la rentabilidad de la empresa (Jensen 1976, 1986; Zeitunet et at., 2017; Modigliani y Miller, 1958, 1963; DeAngelo, H. y Masulis, R. W., 1980; Miller, 1977; Myers, 1984, 2001; Fama y French, 1998; MacKie-Mason, 1990); por lo tanto, las empresas se dividen en las empresas con ROA menor de 6\%, y las empresas con ROA mayor o igual a $6 \%$ (siendo que la mediana es de $6.88 \%$, lo cual se utilizó como referente para dividir los dos grupos).

El análisis estadístico se ejecutó de la siguiente forma: a través de Minitab®, se realizó un estudio de estadística descriptiva, posteriormente se realizaron pruebas ANOVA; y se finalizó con pruebas t. Dado que el interés es conocer el impacto en la estructura de capital después de la crisis del 2008, se analizaron las variables calculadas por año mencionadas en el párrafo anterior por medio de medidas de tendencia central (media) y medidas de dispersión (desviación estándar) tanto del total de las empresas como de cada grupo (ver Apéndice II). Así, se obtuvo el coeficiente de variación (al dividir la desviación estándar entre la media) mismo que permite observar la dispersión de datos con 
respecto a la media.

El análisis de medidas de tendencia central y dispersión sirven como base para realizar una prueba ANOVA. La prueba ANOVA tiene como finalidad establecer si las medias por año de cada una de estas variables no tienen diferencias estadísticamente significativas, es decir, que son iguales entre los años. Para aseverar el hecho de que no existen diferencias estadísticamente significativas se debe comparar el valor p que arroja la prueba con el nivel de significancia para evaluar la hipótesis nula. La hipótesis nula indica que las medias son iguales.

Así, en la Ecuación 1 se establecen la hipótesis nula y la hipótesis alternativa con un nivel de significancia $(\alpha)$ de 0.05 .

Hipótesis Todas las medias son (1) nula iguales

Hipótesis No todas las medias son alternativa iguales

Si $p>\alpha$, indica que no se cuenta con evidencia suficiente para rechazar la hipótesis nula la cual indica que todas las medias son iguales. Si $\mathrm{p} \leq \alpha$, entonces se rechaza la hipótesis nula y se concluye que no todas las medias son iguales. El nivel de significancia $(\alpha)$ utilizado para la prueba ANOVA fue de 0.05 el cual indica un riesgo de 5\% de llegar a la conclusión de que hay una diferencia cuando en realidad no la hay. Para la realización de esta prueba se estableció igualdad de las varianzas ya que se cuenta con 15 empresas (menos de 30) (Levin y Rubin, 2004).

Posteriormente se realizó una prueba $t$ para dos muestras para cada uno de los grupos de empresas, de la media del crecimiento de los activos totales con las variables: a) crecimiento de pasivos totales, b) crecimiento de pasivos circulantes totales, y c) crecimiento de pasivos a largo plazo totales.

La prueba $\mathrm{t}$ pretende determinar si la diferencia entre las medias de las variables es estadísticamente significativa. Dado que se tienen menos de 30 datos, se estableció igualdad de varianzas para este análisis.

Para la prueba t para dos muestras, la hipótesis nula indica que la diferencia entre las variables para cada año no es estadísticamente significativa. En la Ecuación 2 se muestra la hipótesis nula y alternativa para un nivel de significancia $(\alpha)$ de 0.05 .

$$
\begin{aligned}
& H_{0}: \mu_{1}-\mu_{2}=0 \\
& H_{1}: \mu_{1}-\mu_{2} \neq 0
\end{aligned}
$$

Donde $\mu_{1}$ se refiere a la media de la variable de crecimiento de los activos totales; y $\mu_{2}$ se refiere al crecimiento de los pasivos totales, crecimiento de los pasivos a largo plazo totales y crecimiento de los pasivos circulantes totales.

Lo anterior se evalúa comparando el valor $\mathrm{p}$ con respecto al nivel de significancia. En el caso de que $\mathrm{p} \leq \alpha$, se rechaza la hipótesis nula de modo que la diferencia entre las medias es estadísticamente significativa. Si p $>$ a, entonces no se cuenta con suficiente evidencia para rechazar la hipótesis nula, siendo que la diferencia entre las medias no es estadísticamente significativa. El nivel de significancia $(\alpha)$ fue de 0.05 lo que indica un riesgo de $5 \%$ de concluir que existe una diferencia cuando no hay una diferencia real.

\section{RESULTADOS}

\subsection{Estadística descriptiva.}

En esta sección se muestran las medidas de tendencia central para las variables del estudio que son: P/A, PL/A y PC/A. Las medidas de tendencia central y dispersión estudiadas son; media, desviación estándar y coeficiente de variación.

La Figura 1 muestra las medias de P/A (línea a), PL/A (línea b), y PC/A (línea c) para todas las empresas. En la Figura 1 se puede ver cómo el índice de P/A (línea a) de 2007 a 2008 tienen incremento de $41.6 \%$ a $45.6 \%$. (9.5\% incremental), y del año 2008 al 2009 se observa un ligero incremento de $45.6 \%$ a $46.1 \%$ ( $1.2 \%$ incremental).

En las gráficas de las Figuras 2 y 3 se logra ver que la variable de P/A (línea a) incrementa del año 2007 al año 2008. En la Figura 2 se muestran los grupos de empresas con mayor crecimiento en esta variable del año 2007 a 2008. Estos son: activos menores de $\$ 50,000$ millones de pesos (a la izquierda) (de $32.7 \%$ a $39.6 \%$ ) y ROA menor de $6 \%$ (a la derecha) (de $44.3 \%$ a $52.1 \%$ ). Sin embargo, de estos grupos de empresas se puede observar que la variable de PL/A (línea b) 
tuvo un incremento consecutivo del año 2007 al 2008, y del 2008 al 2009. También, se puede observar que PC/A (línea c) tuvieron un incremento en el año 2007 a 2008; sin embargo, se redujo del año 2008 a 2009.

Figura 1. Medias de P/A (línea a), PL/A (línea b), y PC/A (línea c) de la muestra de empresa de los años 2004-2016.

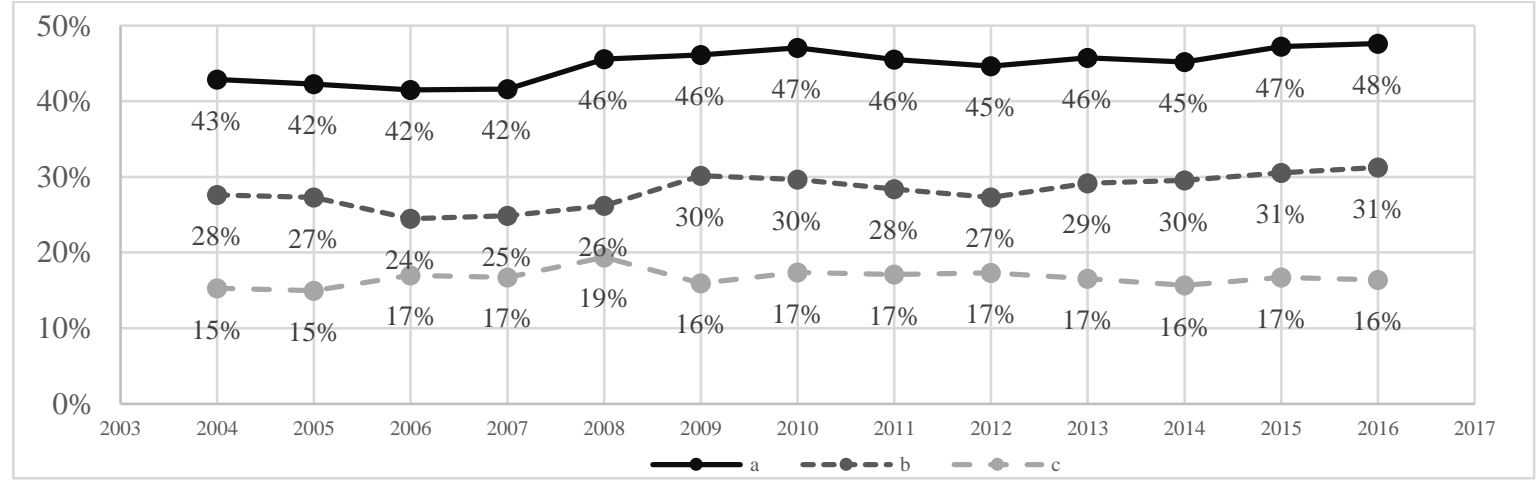

Fuente: Elaboración propia.

Figura 2. Medias de los pasivos totales / activos totales (línea a), pasivos a largo plazo totales / activos totales (línea b), y pasivos circulantes totales / activos totales (línea c) de los años 2004-2016.
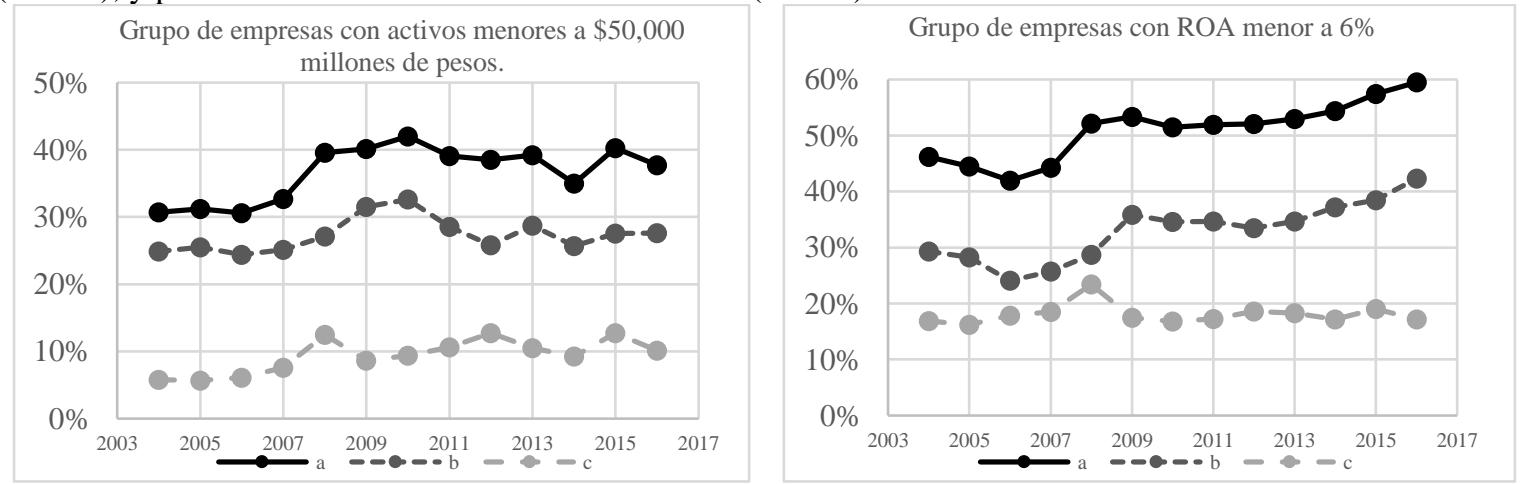

Fuente: Elaboración propia.

Así, en la Figura 3 se aprecia que, para los grupos de empresas de activos mayores o iguales a $\$ 50,0000$ millones de pesos (a la izquierda) y ROA mayor o igual a 6\% (a la derecha), el crecimiento de P/A (línea a) en los años 2007, 2008 y 2009 no es tan pronunciado. Sin embargo, se puede apreciar para este mismo grupo de empresas, que del año 2008 al 2009, la tendencia de la variable de PL/A (línea b) es ascendente, mientras que la de la variable de PC/A (línea c) es descendente.

La Figura 4 muestra la proporción de financiamiento tanto de pasivos a largo plazo como de pasivos a circulantes, ambas con respecto a los pasivos totales. En la Figura 4 se puede notar que en 2007 únicamente el grupo de empresas de activos mayores o iguales a $\$ 50,0000$ millones de pesos tuvo un apalancamiento de $50 \%$ o menos de pasivos a largo plazo con respecto a pasivos totales. Sin embargo, para 2009 todas las empresas superaron el 55\% de los pasivos a largo plazo con respecto a los pasivos totales, siendo que las empresas que tuvieron mayor financiamiento de pasivos a largo plazo fue el grupo de empresas con activos menores a $\$ 50,0000$ millones de pesos (con $73 \%$ ).

Figura 3. Medias de los pasivos totales / activos totales (línea a), pasivos a largo plazo totales / activos totales (línea b), y pasivos circulantes totales / activos totales (línea c) de los años 2004-2016. 


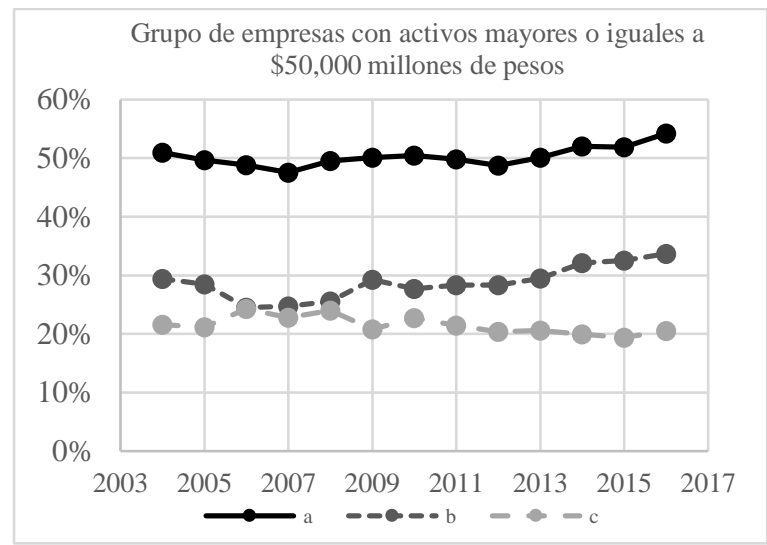

Fuente: Elaboración propia.

Además, en la Figura 4 se puede ver que el grupo de empresas con activos mayores o iguales a $\$ 50,0000$ millones de pesos mantuvo la proporción de pasivos a largo plazo y pasivos circulantes de 2007 a 2008; sin embargo, el grupo incrementó la proporción de pasivos a largo plazo en 2009. Así, del año 2007 al año 2008, las empresas

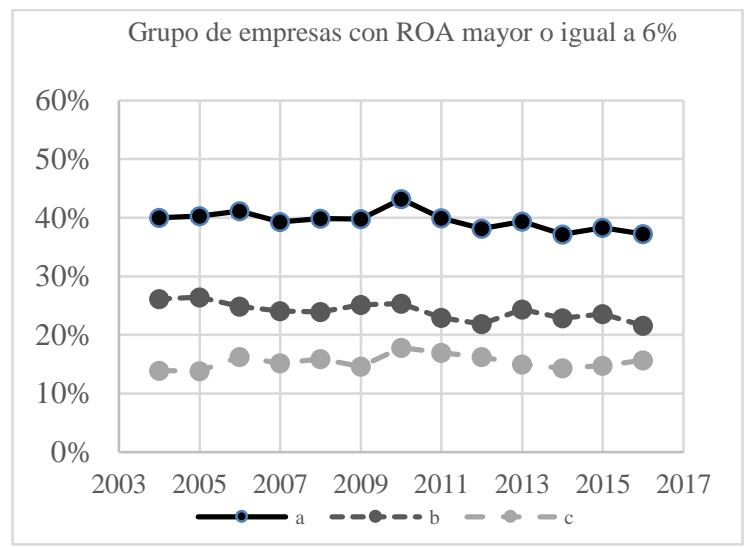

del grupo con activos menores de $\$ 50,000$ millones de pesos incrementó el apalancamiento a través de pasivos circulantes, pero lo redujo nuevamente en 2009. Es importante mencionar que no hubo ningún grupo de empresas que redujeran los pasivos a largo plazo totales con respecto a sus activos totales.

Figura 4. Proporción de financiamiento tanto de pasivos a largo plazo como de pasivos a circulantes con respecto a los pasivos totales.

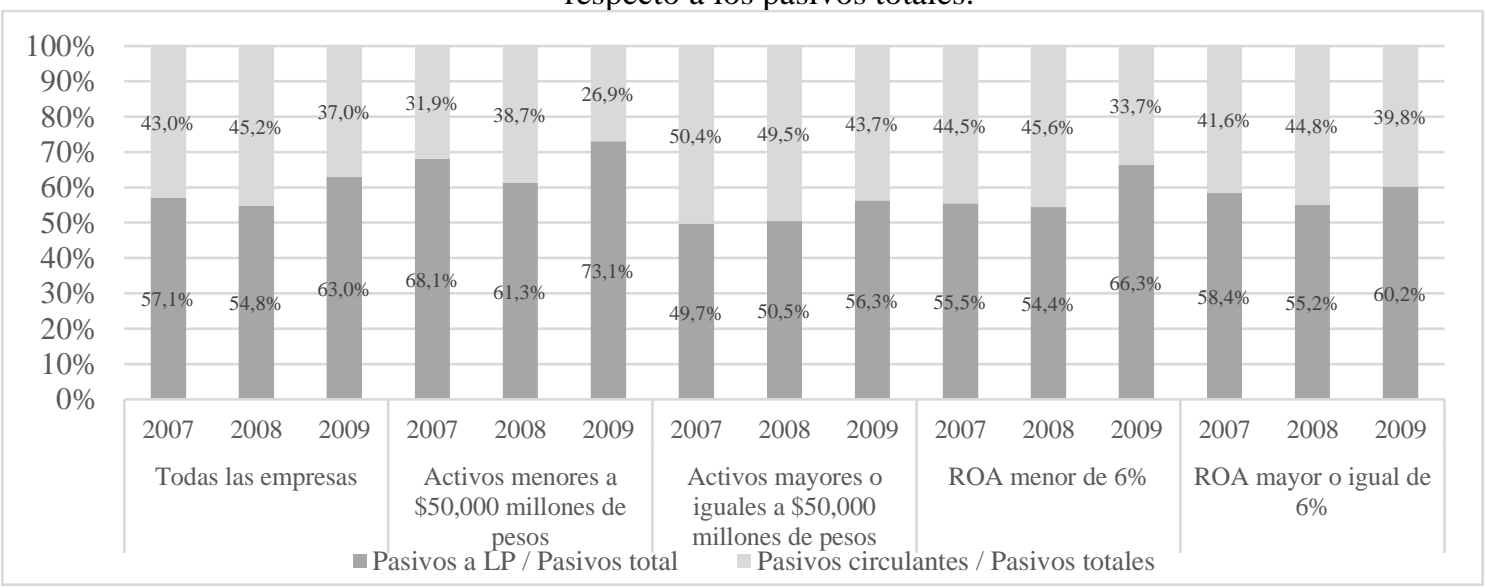

Fuente: Elaboración propia

En el Apéndice II se muestra la estadística descriptiva. Se pueden observar los cambios en las medias de la variable de P/A. Se identifica que del año 2004 al año 2016 el total de empresas muestran que el apalancamiento ha incrementado, siendo que la media del año 2004 fue de $42.88 \%$ y en 2016 incrementó hasta $47.63 \%$ (lo que indica un incremento de $11.1 \%$ ).

Ahora bien, la estadística descriptiva indica que el grupo de empresas de ROA menor de $6 \%$ es el grupo con mayor crecimiento en cuanto a la variable P/A. Este grupo mostró que la media de P/A era de $46.18 \%$ en 2004, mientras que en 2016 mostró un índice de 59.48\%; esto implica un crecimiento de $28.8 \%$ entre los años mencionados. Así, se puede observar que de 2004 a 2008 se tuvo un crecimiento en la variable de $12.9 \%$, de 2008 a 2012 tuvo un cambio de $-0.1 \%$, y de 2012 a 2016, nuevamente creció un $14.2 \%$.

Estos cambios fueron impulsados por el incremento de PL/A. Este grupo mostró un 
índice de PL/A de $29.31 \%$ en 2004 a $42.29 \%$ en 2016 (44.3\% de incremento).

Así mismo, el grupo de empresas con ROA mayor o igual a $6 \%$ muestra un decremento a través de los años (de 2004 a 2016) con respecto a la razón de P/A de $40.00 \%$ a $37.26 \%$ (un cambio de $-6.9 \%$ ).

Ambos grupos de empresas clasificados según el número de activos muestran un crecimiento a través de los años (de 2004 a 2016) con respecto a la razón de $\mathrm{P} / \mathrm{A}$. El grupo con activos mayores o iguales a $\$ 50,000$ millones de pesos tuvo un crecimiento de $4.70 \%$, mientras que el grupo de empresas con activos menores de $\$ 50,000$ millones de pesos tuvo un crecimiento de $21.27 \%$.

En este sentido, es importante mencionar que, del total de empresas, la desviación estándar de P/A se redujo de $24.64 \%$ en 2004 hasta $16.33 \%$ en 2016 , lo que implica un decremento porcentual de 34 puntos. Esto indica que el índice de P/A del total de las empresas se hizo más homogéneo.

Sin embargo, si se observa a detalle la estadística descriptiva de los grupos de empresas, se puede observar el grupo de activos mayores o iguales a $\$ 50,0000$ millones de pesos tiene una desviación estándar creciente a través de los años (del 2004 al 2016) para la variable de P/A. Para 2004, la desviación estándar fue de $7.86 \%$, en cambio para 2016 se incrementó a $14.59 \%$ (un crecimiento de $85.6 \%$ en la desviación estándar). Con todo esto, este grupo se muestra con una desviación estándar aún más baja a la registrada para el total de las empresas en 2016 (16.33\%).

\subsection{Prueba ANOVA de un solo factor para la variable crecimiento de activos totales.}

En la Tabla 3 se muestra la prueba ANOVA de un solo factor para todos los años para las variables del estudio que son: P/A, PL/A y PC/A.

Tabla 3. Resultados de la prueba ANOVA de un solo factor para las variables: a) P/A, b) PL/A, c) PC/A

\begin{tabular}{cccccccc}
\hline & $\boldsymbol{\alpha}$ & $\begin{array}{c}\text { Valor } \\
\boldsymbol{p}(\mathbf{a})\end{array}$ & $\begin{array}{c}\text { Resultado } \\
(\mathbf{a})\end{array}$ & $\begin{array}{c}\text { Valor } \\
\boldsymbol{p}(\mathbf{b})\end{array}$ & $\begin{array}{c}\text { Resultado } \\
(\mathbf{b})\end{array}$ & $\begin{array}{c}\text { Valor } \\
\boldsymbol{p}(\mathbf{c})\end{array}$ & $\begin{array}{c}\text { Resultado } \\
(\mathbf{c})\end{array}$ \\
\hline Todas las empresas & 0.999 & Se acepta $H_{0}$, & 0.998 & Se acepta $H_{0}$, & 0.999 & Se acepta $H_{0}$, \\
lo que & 0.868 & lo que \\
Activos menores de $\$ 50,000$ millones de pesos & & 1.000 & lo que & 1.000 & sue & significa que \\
Activos mayores o iguales a $\$ 50,0000$ millones & 0.05 & & significa que & & significa que & & signifas \\
de pesos & & 0.999 & las medias & 0.982 & las medias son & 0.971 & las medias son \\
ROA menor de 6\% & & 0.937 & son iguales. & 0.597 & iguales. & 0.982 & iguales. \\
ROA mayor o igual a 6\% & 1.000 & & 1.000 & & 1.000 & \\
\hline
\end{tabular}

Fuente: Elaboración propia.

Se puede observar que en todos los casos $\mathrm{p}>\alpha$. Los resultados indican que los valores de las medias de las variables del estudio, para los 13 años, no muestran diferencias estadísticamente significativas. Por lo tanto, no se puede rechazar la hipótesis nula que indica que todas las medias son iguales.

Es importante mencionar que, aunque no se tiene evidencia para rechazar la hipótesis nula, la cual indica que las medias son iguales, la estadística descriptiva muestra cambios notorios en del año 2007 al año 2008 y del año 2008 al 2009. Así mismo, los coeficientes de variación indican que la desviación estándar representa entre $20 \%$ y $100 \%$ con respecto a la media (según el grupo de empresas y la variable), lo que demuestra que los datos son muy dispersos.
4.3. Prueba $t$ para las variables de crecimiento de activos totales con crecimiento de pasivos totales, crecimiento de pasivos a largo plazo totales $y$ crecimiento de pasivos circulantes totales.

En la Tabla 4 se muestra el resultado de la prueba $t$ de dos muestras para el crecimiento únicamente de los años 2008 y 2009. Se omitieron el resto de los años pues, como se muestra en la estadística descriptiva del Apéndice II y en las Figuras 1, 2 y 3, se observan cambios notorios del año 2007 al año 2008 y del año 2008 al 2009. Se establece que $\mu_{1}$ se refiere a la media del crecimiento de los pasivos totales, pasivos a largo plazo totales y pasivos circulantes totales; y $\mu_{2}$ es la media de los activos totales. 
Tabla 4. Resultados de prueba t de dos muestras para los años 2008 y 2009 para: a) las variables de crecimiento de activos totales y crecimiento de deuda total, b) las variables de crecimiento de activos totales y crecimiento de pasivos a largo plazo totales, y c) las variables de crecimiento de activos totales y crecimiento de pasivos circulantes totales.

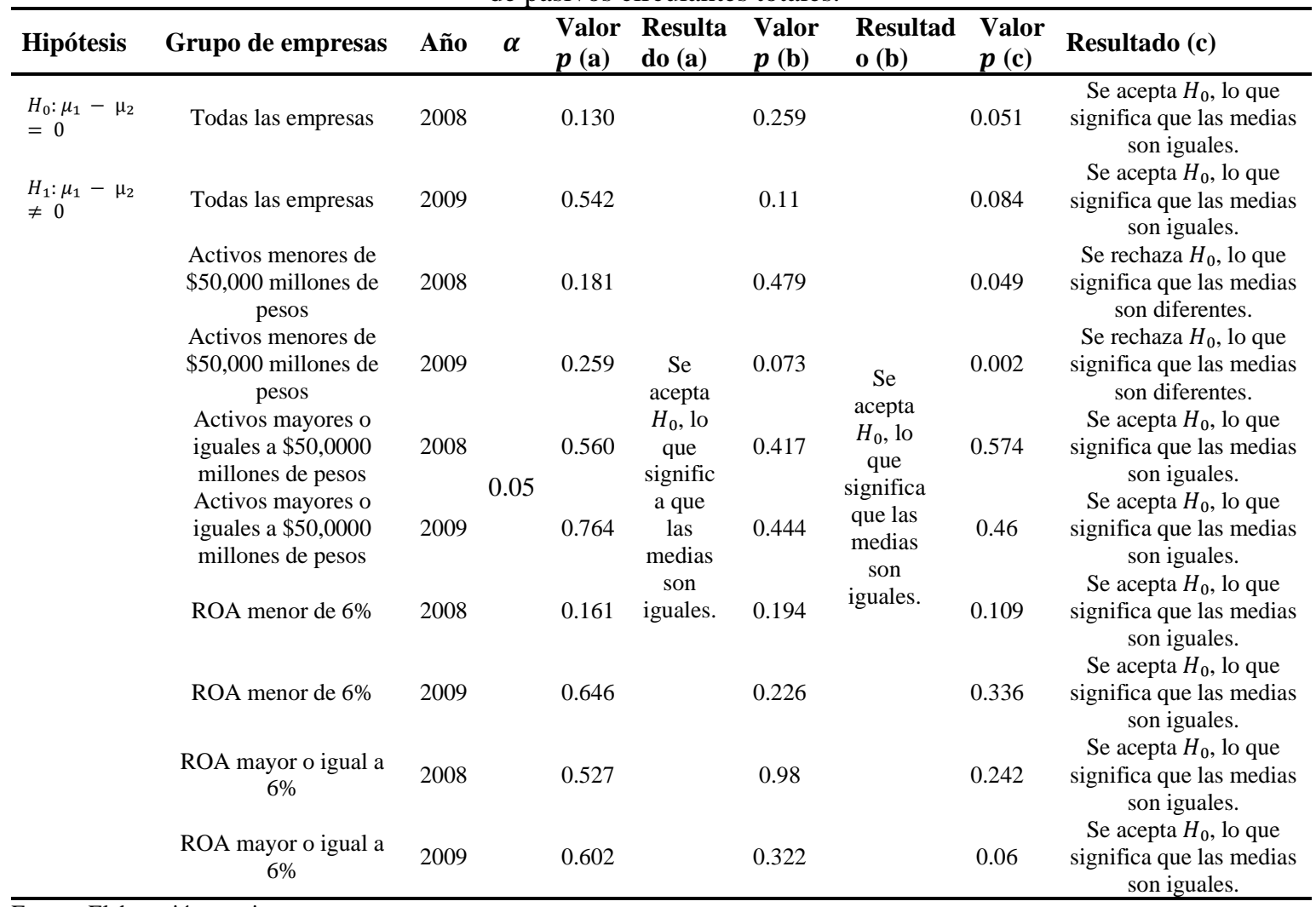

Fuente: Elaboración propia

En todos los grupos de empresas para ambos años (a excepción del grupo de activos menores de $\$ 50,000$ millones de pesos), el valor $\mathrm{p}>\alpha$, por lo que, si bien se observan diferencias en las medias, la evidencia de las pruebas de hipótesis t señalan que estas diferencias no son estadísticamente significativas, lo que permite establecer, que las medias son iguales.

Según lo observado en la Tabla 4, para las medias del crecimiento de los pasivos circulantes totales y del crecimiento de los activos totales, el único grupo de empresas en el que se rechaza la hipótesis nula es el de activos menores de $\$ 50,000$ millones de pesos (para ambos años). Por lo tanto, se asume que para este grupo las diferencias de las medias del crecimiento de los pasivos circulantes totales y del crecimiento de los activos totales son estadísticamente significativas, por lo tanto, las medias son diferentes.

\subsection{Discusión teórica de los resultados.}

No se observa una diferencia estadísticamente significativa entre las medias de los años 2004 a 2016 después de aplicar pruebas ANOVA para cada una de las siguientes variables: P/A, PL/A y PC/A. Sin embargo, en el análisis de la estadística descriptiva se encontró que el apalancamiento general incrementó durante la crisis hipotecaria del 2008 (de 2007 a 2009) para todas las empresas (ver sección 4.1. y Apéndice II ). Sin embargo, la distribución de los pasivos a largo plazo y circulantes no incrementó en la misma proporción. De 2007 a 2008, el apalancamiento a corto plazo incrementó en mayor proporción que el de largo plazo; lo cual indica que, además de las empresas recurrieron a mayor financiamiento, parte del financiamiento a largo plazo fue sustituido por financiamiento a corto plazo. Sin embargo, de 2008 a 2009, el apalancamiento de corto plazo vuelve a disminuir y se incrementa el financiamiento por medio de pasivos a largo plazo (ver Figura 4). Estos resultados coinciden 
con algunos autores (Alves y Francisco, 2015; Christensen et al., 2015; Lopes, 2017; Alves y Francisco, 2015; Puntaier, 2010; González, 2014; Custódio et al., 2013) que indican que, en tiempos de crisis, el apalancamiento incrementa y que la deuda a largo plazo es sustituida por deuda a corto plazo.

En el Apéndice II, también se puede observar que el apalancamiento de 2004 a 2016 incrementó; sin embargo, por medio de la desviación estándar y el coeficiente de variación se puede analizar que los datos de empresas para las variables del estudio se hicieron más homogéneos. Esto indica que las empresas, con el paso del tiempo, siguieron la tendencia de mantener un apalancamiento similar.

A pesar de los cambios expuestos anteriormente, no se observa una diferencia estadísticamente significativa entre las medias de los años 2004 a 2016 después de aplicar las pruebas ANOVA para cada una de las variables del estudio. Esto indica que a lo largo de los 13 años estudiados (2004-2016) el apalancamiento general fue creciente pero paulatino $y$ sin cambios drásticos.

Es importante mencionar que, para el grupo de empresas con activos menores de $\$ 50,000$ millones de pesos, se encontró que la media del crecimiento de PC/A no es la misma que el crecimiento de los activos. Esto indica que los pasivos circulantes no tuvieron el mismo ritmo de crecimiento que los activos totales (ver Tabla 4). En la Figura 4, se observa cómo la proporción de pasivos circulantes del total de pasivos cambia notoriamente del año 2007 al 2008 y del año 2008 al 2009. Como lo explica Modigliani y Miller (1958), la inversión de capital (que incrementa la cuenta de activos en el balance general) tiene como finalidad incrementar las ventas y maximizar el rendimiento para los accionistas (que se puede medir a través del ROA).

\section{CONCLUSIONES}

El presente estudio tiene la finalidad de identificar el impacto de la crisis hipotecaria financiera del 2008 en la estructura de capital en las empresas públicas mexicanas listadas en el Índice de Precios y Cotizaciones (IPC) de la Bolsa Mexicana de Valores (BMV).

Los resultados del estudio indican que durante la llamada crisis hipotecaria del 2008 el apalancamiento general incrementó de 2007 a 2009. Se observa que en el primer año de la crisis (de 2007 a 2008) el apalancamiento por medio de pasivos circulantes incrementó en mayor proporción que el financiamiento por medio de pasivos a largo plazo. Sin embargo, en el segundo año (de 2008 a 2009), el apalancamiento a través de pasivos circulantes se redujo de forma tal que éste fue sustituido por deuda a largo plazo. Estos resultados coinciden con algunos como Christensen et al. (2015), Lopes (2017), González (2014), Alves y Francisco (2015), Puntaier (2010) y Custódio et al. (2013).

El análisis del año 2004 al 2016, además de que el apalancamiento general se incrementó, los resultados indican que el incremento fue paulatino, además de que la razón de apalancamiento de las empresas se homogenizó a lo largo de los años.

Se observó que, únicamente para el grupo de empresas con activos menores de $\$ 50,000$ millones de pesos el crecimiento de activos totales no fue el mismo que el crecimiento de PC/A.

Por otra parte, una de las limitantes del estudio es que la muestra fue de 15 empresas, siendo que el IPC representa 35 empresas, de las cuales fueron eliminadas las que operan en el sector financiero y las que en alguno de los años entre el año 2004 y 2016 no cotizaron en la BMV.

Dados los resultados de este estudio, se proponen 4 líneas de investigación. La primera es estudiar si para las empresas con activos menores a $\$ 50,000$ millones de pesos las empresas con mayor ROA difieren de las que tienen mejor ROA en cuanto a la sensibilidad de la sustitución de deuda a largo plazo por deuda a corto plazo y viceversa en tiempos de crisis. La segunda línea de investigación es identificar las causas por las cuales el apalancamiento de las empresas se homogenizó a lo largo de los años, las razones por las cuales las empresas optan por tener un determinado apalancamiento, y si existe una causa coincidente entre las empresas para mantenerse en un determinado rango de apalancamiento. La tercera línea de investigación es el estudiar si el apalancamiento cambia por tipo de industria en los años de 2007 
a 2009. Y finalmente, se podría estudiar si los resultados de este estudio son coincidentes con el apalancamiento de la crisis económica de 2020 .
Con base en lo anterior, se establece que el objetivo del presente trabajo se ha cumplido a cabalidad.

\section{APÉNDICE}

Apéndice I. Empresas de las cuales se obtuvo información de la base de datos de Standard \& Poors (2021).

\section{Empresa}

Alfa, S. A. B. de C. V.

Arca Continental, S.A.B. de C.V.

CEMEX, S.A.B. de C.V.

El Puerto de Liverpool, S.A.B. de C.V.

Fomento Económico Mexicano, S.A.B. de C.V.

Gruma, S.A.B. de C.V.

Grupo Aeroportuario del Centro Norte, S.A.B. de C.V.

Grupo Aeroportuario del Pacífico, S.A.B. de C.V.

Grupo Aeroportuario del Sureste, S. A. B. de C. V.

Grupo Bimbo, S.A.B. de C.V.

Grupo Carso, S.A.B. de C.V.

Grupo Televisa, S.A.B.

Industrias Peñoles, S.A.B. de C.V.

Promotora y Operadora de Infraestructura, S. A. B. de C. V.

Wal-Mart de México, S.A.B. de C.V.

Fuente: Elaboración propia.

Apéndice II. Estadística descriptiva de todas las empresas de las variables de pasivos totales / activos totales, pasivos a largo plazo / activos totales, y pasivos circulantes / activos totales.

\begin{tabular}{|c|c|c|c|c|c|c|c|c|c|}
\hline & \multicolumn{3}{|c|}{$\begin{array}{l}\text { Pasivos totales / } \\
\text { activos totales }\end{array}$} & \multicolumn{3}{|c|}{$\begin{array}{c}\text { Pasivos a largo plazo / } \\
\text { activos totales }\end{array}$} & \multicolumn{3}{|c|}{$\begin{array}{l}\text { Pasivos circulantes / } \\
\text { activos totales }\end{array}$} \\
\hline Año & Media & Desv.Est. & $\begin{array}{r}\text { Coef. de } \\
\text { var. }\end{array}$ & Media & Desv.Est. & $\begin{array}{l}\text { Coef. de } \\
\text { var. }\end{array}$ & Media & Desv.Est. & $\begin{array}{r}\text { Coef. de } \\
\text { var. }\end{array}$ \\
\hline 2004 & $42.88 \%$ & $24.64 \%$ & $57.46 \%$ & $27.60 \%$ & $21.52 \%$ & $77.97 \%$ & $15.28 \%$ & $10.35 \%$ & $67.74 \%$ \\
\hline 2005 & $42.26 \%$ & $23.79 \%$ & $56.29 \%$ & $27.29 \%$ & $21.24 \%$ & $77.83 \%$ & $14.97 \%$ & $9.62 \%$ & $64.26 \%$ \\
\hline 2006 & $41.50 \%$ & $22.88 \%$ & $55.13 \%$ & $24.48 \%$ & $20.21 \%$ & $82.56 \%$ & $17.02 \%$ & $10.85 \%$ & $63.75 \%$ \\
\hline 2007 & $41.61 \%$ & $21.02 \%$ & $50.52 \%$ & $24.87 \%$ & $20.14 \%$ & $80.98 \%$ & $16.74 \%$ & $9.41 \%$ & $56.21 \%$ \\
\hline 2008 & $45.56 \%$ & $22.49 \%$ & $49.36 \%$ & $26.18 \%$ & $19.41 \%$ & $74.14 \%$ & $19.38 \%$ & $10.48 \%$ & $54.08 \%$ \\
\hline 2009 & $46.10 \%$ & $21.06 \%$ & $45.68 \%$ & $30.14 \%$ & $19.99 \%$ & $66.32 \%$ & $15.97 \%$ & $9.35 \%$ & $58.55 \%$ \\
\hline 2010 & $47.05 \%$ & $19.93 \%$ & $42.36 \%$ & $29.68 \%$ & $19.66 \%$ & $66.24 \%$ & $17.38 \%$ & $12.21 \%$ & $70.25 \%$ \\
\hline 2011 & $45.52 \%$ & $19.79 \%$ & $43.48 \%$ & $28.41 \%$ & $18.71 \%$ & $65.86 \%$ & $17.11 \%$ & $9.71 \%$ & $56.75 \%$ \\
\hline 2012 & $44.64 \%$ & $18.71 \%$ & $41.91 \%$ & $27.31 \%$ & $16.26 \%$ & $59.54 \%$ & $17.33 \%$ & $10.09 \%$ & $58.22 \%$ \\
\hline 2013 & $45.72 \%$ & $16.38 \%$ & $35.83 \%$ & $29.17 \%$ & $14.58 \%$ & $49.98 \%$ & $16.55 \%$ & $9.28 \%$ & $56.07 \%$ \\
\hline 2014 & $45.21 \%$ & $17.90 \%$ & $39.59 \%$ & $29.56 \%$ & $15.91 \%$ & $53.82 \%$ & $15.66 \%$ & $8.55 \%$ & $54.60 \%$ \\
\hline 2015 & $47.25 \%$ & $16.21 \%$ & $34.31 \%$ & $30.52 \%$ & $15.59 \%$ & $51.08 \%$ & $16.73 \%$ & $8.01 \%$ & $47.88 \%$ \\
\hline 2016 & $47.63 \%$ & $16.33 \%$ & $34.29 \%$ & $31.25 \%$ & $15.81 \%$ & $50.59 \%$ & $16.38 \%$ & $9.45 \%$ & $57.69 \%$ \\
\hline \multicolumn{10}{|c|}{ a) Activos menores de $\$ 50,000$ millones de pesos } \\
\hline & \multicolumn{3}{|c|}{$\begin{array}{c}\text { Pasivos totales / } \\
\text { activos totales }\end{array}$} & \multicolumn{3}{|c|}{$\begin{array}{l}\text { Pasivos a largo plazo / } \\
\text { activos totales }\end{array}$} & \multicolumn{3}{|c|}{$\begin{array}{l}\text { Pasivos circulantes / } \\
\text { activos totales }\end{array}$} \\
\hline Año & Media & Desv.Est. & $\begin{array}{r}\text { Coef. de } \\
\text { var. }\end{array}$ & Media & Desv.Est. & $\begin{array}{r}\text { Coef. de } \\
\text { var. }\end{array}$ & Media & Desv.Est. & $\begin{array}{r}\text { Coef. de } \\
\text { var. }\end{array}$ \\
\hline 2004 & $30.70 \%$ & $36.10 \%$ & $117.59 \%$ & $24.90 \%$ & $33.30 \%$ & $133.73 \%$ & $5.80 \%$ & $5.42 \%$ & $93.45 \%$ \\
\hline 2005 & $31.20 \%$ & $35.40 \%$ & $113.46 \%$ & $25.50 \%$ & $33.00 \%$ & $129.41 \%$ & $5.69 \%$ & $5.32 \%$ & $93.50 \%$ \\
\hline 2006 & $30.60 \%$ & $34.40 \%$ & $112.42 \%$ & $24.40 \%$ & $32.00 \%$ & $131.15 \%$ & $6.12 \%$ & $6.21 \%$ & $101.47 \%$ \\
\hline 2007 & $32.70 \%$ & $30.40 \%$ & $92.97 \%$ & $25.10 \%$ & $30.10 \%$ & $119.92 \%$ & $7.61 \%$ & $7.24 \%$ & $95.14 \%$ \\
\hline 2008 & $39.60 \%$ & $33.60 \%$ & $84.85 \%$ & $27.10 \%$ & $30.10 \%$ & $111.07 \%$ & $12.48 \%$ & $12.53 \%$ & $100.40 \%$ \\
\hline 2009 & $40.10 \%$ & $30.80 \%$ & $76.81 \%$ & $31.50 \%$ & $28.80 \%$ & $91.43 \%$ & $8.66 \%$ & $7.79 \%$ & $89.95 \%$ \\
\hline 2010 & $42.00 \%$ & $28.20 \%$ & $67.14 \%$ & $32.60 \%$ & $25.40 \%$ & $77.91 \%$ & $9.36 \%$ & $8.21 \%$ & $87.71 \%$ \\
\hline 2011 & $39.10 \%$ & $24.60 \%$ & $62.92 \%$ & $28.51 \%$ & $20.08 \%$ & $70.43 \%$ & $10.61 \%$ & $7.52 \%$ & $70.88 \%$ \\
\hline 2012 & $38.52 \%$ & $23.80 \%$ & $61.79 \%$ & $25.79 \%$ & $16.33 \%$ & $63.32 \%$ & $12.73 \%$ & $11.67 \%$ & $91.67 \%$ \\
\hline 2013 & $39.20 \%$ & $19.53 \%$ & $49.82 \%$ & $28.72 \%$ & $13.88 \%$ & $48.33 \%$ & $10.48 \%$ & $8.51 \%$ & $81.20 \%$ \\
\hline 2014 & $34.96 \%$ & $16.66 \%$ & $47.65 \%$ & $25.70 \%$ & $12.89 \%$ & $50.16 \%$ & $9.26 \%$ & $7.11 \%$ & $76.78 \%$ \\
\hline 2015 & $40.25 \%$ & $15.19 \%$ & $37.74 \%$ & $27.52 \%$ & $11.61 \%$ & $42.19 \%$ & $12.73 \%$ & $8.28 \%$ & $65.04 \%$ \\
\hline
\end{tabular}




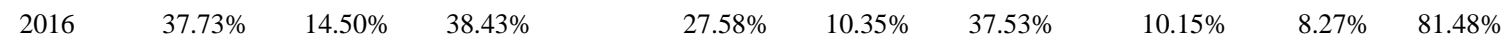

\begin{tabular}{|c|c|c|c|c|c|c|c|c|c|}
\hline \multicolumn{10}{|c|}{ b) Activos mayores o iguales a $\$ 50,0000$ millones de pesos } \\
\hline & \multicolumn{3}{|c|}{$\begin{array}{l}\text { Pasivos totales / } \\
\text { activos totales }\end{array}$} & \multicolumn{3}{|c|}{$\begin{array}{l}\text { Pasivos a largo plazo / } \\
\text { activos totales }\end{array}$} & \multicolumn{3}{|c|}{$\begin{array}{l}\text { Pasivos circulantes / } \\
\text { activos totales }\end{array}$} \\
\hline Año & Media & Desv.Est. & $\begin{array}{l}\text { Coef. de } \\
\text { var. }\end{array}$ & Media & Desv.Est. & $\begin{array}{l}\text { Coef. de } \\
\text { var. }\end{array}$ & Media & Desv.Est. & $\begin{array}{l}\text { Coef. de } \\
\text { var. }\end{array}$ \\
\hline 2004 & $50.99 \%$ & $7.86 \%$ & $15.41 \%$ & $29.39 \%$ & $10.50 \%$ & $35.73 \%$ & $21.60 \%$ & $7.53 \%$ & $34.86 \%$ \\
\hline 2005 & $49.65 \%$ & $7.31 \%$ & $14.72 \%$ & $28.50 \%$ & $10.22 \%$ & $35.86 \%$ & $21.15 \%$ & $6.08 \%$ & $28.75 \%$ \\
\hline 2006 & $48.79 \%$ & $5.31 \%$ & $10.88 \%$ & $24.51 \%$ & $8.73 \%$ & $35.62 \%$ & $24.28 \%$ & $5.79 \%$ & $23.85 \%$ \\
\hline 2007 & $47.56 \%$ & $9.82 \%$ & $20.65 \%$ & $24.74 \%$ & $11.96 \%$ & $48.34 \%$ & $22.82 \%$ & $4.27 \%$ & $18.71 \%$ \\
\hline 2008 & $49.54 \%$ & $11.52 \%$ & $23.25 \%$ & $25.56 \%$ & $9.61 \%$ & $37.60 \%$ & $23.99 \%$ & $5.87 \%$ & $24.47 \%$ \\
\hline 2009 & $50.08 \%$ & $11.82 \%$ & $23.60 \%$ & $29.24 \%$ & $13.40 \%$ & $45.83 \%$ & $20.83 \%$ & $6.95 \%$ & $33.37 \%$ \\
\hline 2010 & $50.44 \%$ & $12.83 \%$ & $25.44 \%$ & $27.72 \%$ & $16.19 \%$ & $58.41 \%$ & $22.72 \%$ & $11.76 \%$ & $51.76 \%$ \\
\hline 2011 & $49.79 \%$ & $16.03 \%$ & $32.20 \%$ & $28.34 \%$ & $18.98 \%$ & $66.97 \%$ & $21.45 \%$ & $8.75 \%$ & $40.79 \%$ \\
\hline 2012 & $48.72 \%$ & $14.54 \%$ & $29.84 \%$ & $28.33 \%$ & $17.11 \%$ & $60.40 \%$ & $20.40 \%$ & $8.16 \%$ & $40.00 \%$ \\
\hline 2013 & $50.07 \%$ & $13.34 \%$ & $26.64 \%$ & $29.47 \%$ & $15.86 \%$ & $53.82 \%$ & $20.60 \%$ & $7.71 \%$ & $37.43 \%$ \\
\hline 2014 & $52.04 \%$ & $16.01 \%$ & $30.76 \%$ & $32.12 \%$ & $17.91 \%$ & $55.76 \%$ & $19.92 \%$ & $6.73 \%$ & $33.79 \%$ \\
\hline 2015 & $51.91 \%$ & $15.94 \%$ & $30.71 \%$ & $32.52 \%$ & $18.17 \%$ & $55.87 \%$ & $19.40 \%$ & $7.03 \%$ & $36.24 \%$ \\
\hline 2016 & $54.23 \%$ & $14.59 \%$ & $26.90 \%$ & $33.70 \%$ & $18.80 \%$ & $55.79 \%$ & $20.53 \%$ & $8.05 \%$ & $39.21 \%$ \\
\hline
\end{tabular}

\begin{tabular}{|c|c|c|c|c|c|c|c|c|c|}
\hline \multicolumn{10}{|c|}{ c) ROA menor de $6 \%$} \\
\hline & \multicolumn{3}{|c|}{$\begin{array}{c}\text { Pasivos totales / } \\
\text { activos totales }\end{array}$} & \multicolumn{3}{|c|}{$\begin{array}{c}\text { Pasivos a largo plazo / } \\
\text { activos totales }\end{array}$} & \multicolumn{3}{|c|}{$\begin{array}{c}\text { Pasivos circulantes / } \\
\text { activos totales }\end{array}$} \\
\hline Año & Media & Desv.Est. & $\begin{array}{r}\text { Coef. de } \\
\text { var. }\end{array}$ & Media & Desv.Est. & $\begin{array}{r}\text { Coef. de } \\
\text { var. }\end{array}$ & Media & Desv.Est. & $\begin{array}{l}\text { Coef. de } \\
\text { var. }\end{array}$ \\
\hline 2004 & $46.18 \%$ & $20.74 \%$ & $44.91 \%$ & $29.31 \%$ & $13.29 \%$ & $45.34 \%$ & $16.87 \%$ & $9.33 \%$ & $55.31 \%$ \\
\hline 2005 & $44.49 \%$ & $20.17 \%$ & $45.34 \%$ & $28.25 \%$ & $13.72 \%$ & $48.57 \%$ & $16.23 \%$ & $8.52 \%$ & $52.50 \%$ \\
\hline 2006 & $41.95 \%$ & $18.44 \%$ & $43.96 \%$ & $24.07 \%$ & $11.88 \%$ & $49.36 \%$ & $17.88 \%$ & $9.06 \%$ & $50.67 \%$ \\
\hline 2007 & $44.28 \%$ & $19.77 \%$ & $44.65 \%$ & $25.75 \%$ & $14.58 \%$ & $56.62 \%$ & $18.53 \%$ & $8.12 \%$ & $43.82 \%$ \\
\hline 2008 & $52.12 \%$ & $24.38 \%$ & $46.78 \%$ & $28.72 \%$ & $14.18 \%$ & $49.37 \%$ & $23.40 \%$ & $10.79 \%$ & $46.11 \%$ \\
\hline 2009 & $53.34 \%$ & $22.46 \%$ & $42.11 \%$ & $35.83 \%$ & $16.29 \%$ & $45.46 \%$ & $17.51 \%$ & $8.75 \%$ & $49.97 \%$ \\
\hline 2010 & $51.45 \%$ & $23.42 \%$ & $45.52 \%$ & $34.61 \%$ & $17.20 \%$ & $49.70 \%$ & $16.84 \%$ & $8.11 \%$ & $48.16 \%$ \\
\hline 2011 & $51.91 \%$ & $22.65 \%$ & $43.63 \%$ & $34.64 \%$ & $18.15 \%$ & $52.40 \%$ & $17.27 \%$ & $7.45 \%$ & $43.14 \%$ \\
\hline 2012 & $52.08 \%$ & $22.31 \%$ & $42.84 \%$ & $33.50 \%$ & $17.47 \%$ & $52.15 \%$ & $18.58 \%$ & $9.65 \%$ & $51.94 \%$ \\
\hline 2013 & $52.99 \%$ & $20.84 \%$ & $39.33 \%$ & $34.66 \%$ & $15.69 \%$ & $45.27 \%$ & $18.33 \%$ & $8.34 \%$ & $45.50 \%$ \\
\hline 2014 & $54.39 \%$ & $21.81 \%$ & $40.10 \%$ & $37.20 \%$ & $17.17 \%$ & $46.16 \%$ & $17.19 \%$ & $6.41 \%$ & $37.29 \%$ \\
\hline 2015 & $57.44 \%$ & $16.05 \%$ & $27.94 \%$ & $38.44 \%$ & $14.90 \%$ & $38.76 \%$ & $19.00 \%$ & $4.87 \%$ & $25.63 \%$ \\
\hline 2016 & $59.48 \%$ & $13.02 \%$ & $21.89 \%$ & $42.29 \%$ & $10.67 \%$ & $25.23 \%$ & $17.19 \%$ & $6.65 \%$ & $38.69 \%$ \\
\hline
\end{tabular}

\begin{tabular}{|c|c|c|c|c|c|c|c|c|c|}
\hline \multicolumn{10}{|c|}{ d) ROA mayor o igual a $6 \%$} \\
\hline & \multicolumn{3}{|c|}{$\begin{array}{l}\text { Pasivos totales / } \\
\text { activos totales }\end{array}$} & \multicolumn{3}{|c|}{$\begin{array}{c}\text { Pasivos a largo plazo / } \\
\text { activos totales }\end{array}$} & \multicolumn{3}{|c|}{$\begin{array}{c}\text { Pasivos circulantes / } \\
\text { activos totales }\end{array}$} \\
\hline Año & Media & Desv.Est. & $\begin{array}{r}\text { Coef. de } \\
\text { var. }\end{array}$ & Media & Desv.Est. & $\begin{array}{r}\text { Coef. de } \\
\text { var. }\end{array}$ & Media & Desv.Est. & $\begin{array}{r}\text { Coef. de } \\
\text { var. }\end{array}$ \\
\hline 2004 & $40.00 \%$ & $28.70 \%$ & $71.75 \%$ & $26.11 \%$ & $27.74 \%$ & $106.24 \%$ & $13.89 \%$ & $11.61 \%$ & $83.59 \%$ \\
\hline 2005 & $40.31 \%$ & $27.82 \%$ & $69.02 \%$ & $26.45 \%$ & $27.20 \%$ & $102.84 \%$ & $13.86 \%$ & $10.96 \%$ & $79.08 \%$ \\
\hline 2006 & $41.11 \%$ & $27.47 \%$ & $66.82 \%$ & $24.85 \%$ & $26.37 \%$ & $106.12 \%$ & $16.26 \%$ & $12.80 \%$ & $78.72 \%$ \\
\hline 2007 & $39.26 \%$ & $23.13 \%$ & $58.91 \%$ & $24.09 \%$ & $25.05 \%$ & $103.99 \%$ & $15.17 \%$ & $10.71 \%$ & $70.60 \%$ \\
\hline 2008 & $39.83 \%$ & $20.52 \%$ & $51.52 \%$ & $23.96 \%$ & $23.86 \%$ & $99.58 \%$ & $15.87 \%$ & $9.47 \%$ & $59.67 \%$ \\
\hline 2009 & $39.77 \%$ & $18.89 \%$ & $47.50 \%$ & $25.16 \%$ & $22.61 \%$ & $89.86 \%$ & $14.61 \%$ & $10.23 \%$ & $70.02 \%$ \\
\hline 2010 & $43.20 \%$ & $16.96 \%$ & $39.26 \%$ & $25.36 \%$ & $21.76 \%$ & $85.80 \%$ & $17.84 \%$ & $15.53 \%$ & $87.05 \%$ \\
\hline 2011 & $39.93 \%$ & $16.34 \%$ & $40.92 \%$ & $22.95 \%$ & $18.57 \%$ & $80.92 \%$ & $16.97 \%$ & $11.86 \%$ & $69.89 \%$ \\
\hline 2012 & $38.14 \%$ & $13.02 \%$ & $34.14 \%$ & $21.90 \%$ & $13.97 \%$ & $63.79 \%$ & $16.24 \%$ & $10.98 \%$ & $67.61 \%$ \\
\hline 2013 & $39.36 \%$ & $8.07 \%$ & $20.50 \%$ & $24.37 \%$ & $12.56 \%$ & $51.54 \%$ & $15.00 \%$ & $10.34 \%$ & $68.93 \%$ \\
\hline 2014 & $37.18 \%$ & $8.68 \%$ & $23.35 \%$ & $22.87 \%$ & $12.00 \%$ & $52.47 \%$ & $14.32 \%$ & $10.33 \%$ & $72.14 \%$ \\
\hline 2015 & $38.33 \%$ & $10.47 \%$ & $27.32 \%$ & $23.59 \%$ & $13.36 \%$ & $56.63 \%$ & $14.74 \%$ & $9.91 \%$ & $67.23 \%$ \\
\hline 2016 & $37.26 \%$ & $11.17 \%$ & $29.98 \%$ & $21.59 \%$ & $13.18 \%$ & $61.05 \%$ & $15.66 \%$ & $11.80 \%$ & $75.35 \%$ \\
\hline
\end{tabular}

Fuente: Elaboración propia 


\section{REFERENCIAS}

Alves, P., Francisco, P. (2015). The impact of institutional environment on the capital structure of firms during recent financial crises. The Quarterly Review of Economics and Finance, 57, 129-146.

Arias, M., A. L., Fierro, F., Pelayo, M., y Cobián, S. (2009). Factores institucionales que influyen en la decisión de estructura de capital de las empresas en México. Expresión Económica, 49-64.

Booth, L., Aivazian, V., Demirguc-Kunt, A., y Maksimovic, V. (2001). Capital structures in developing countries. The journal of finance, 56(1), 87-130.

Calderón, C. y Bielma, L. (2017). Integración económica, crisis económicas y ciclos económicos en México. Contaduría y administración, 62(1), 64-84.

Chen, S., Ganum, P., Liu, L., Martinez, L., Martinez S., (2018). Debt Maturity and the Use of Short-term Debt: Evidence from Sovereigns and Firms, International Monetary Fund. Washington, D.C.: Fondo Monetario Internacional.

Christensen, B. J., Nielsen, M., Zhu, J. (2015). The impact of financial crises on the risk-return tradeoff and the leverage effect. Economic Modelling, 49, 407-418.

Correa, E. (2010). México, crisis económica y financiera. Ola Financiera, 3(6).

Custódio, C., Ferreira, M. A., Laureano, L. (2013). Why are US firms using more short-term debt?. Journal of Financial Economics, 108(1), 182-212.

DeAngelo, H. y Masulis, R. W. (1980). Optimal capital structure under corporate and personal taxation. Journal of Financial Economics, 8(1), 3-29.

Donaldson, G. (1961). Corporate debt capacity, Harvard. Harvard University Press. Downs, TW,(1991).“An alternate approach to fundamental analysis: The asset side of the equation.” Journal of Portfolio Management, 17(2), 6-17.

Fama E.F. y French K.R., (1998) Taxes, financing decisions, and firm value, The Journal of Finance, 53(3), 819-843 (1998).

González, V. M. (2014). The financial crisis and corporate debt maturity. Available at SSRN 2502798.

INSTITUTO MEXICANO DE CONTADORES PUBLICOS (2014). Normas de Información Financiera 2014 (NIF). Boletín B-14, utilidad por acción. México. IMCP. Novena edición.

Jensen, M. C. (1986). Agency Costs of Free Cash Flow, Corporate Finance, and Takeovers. The American Economic Review, 76(2), 323-329.

Jensen, M. C., y Meckling, W. H. (1976). Theory of the firm: Managerial behavior, agency costs and ownership structure. Journal of Financial Economics, 3(4), 305-360.

Levin, R, y Rubin, D. (2004). Estadística para administración y economía. Pearson Educación.

Lopes, N. (2017). The Determinants of Capital Structure: impact of institutional determinants and subprime crisis.

MacKie-Mason J.K. (1990). Do taxes affect corporate financing decisions? The Journal of Finance, 45(5), 1471-1493.

Miller, M. H. (1977). Debt and taxes. The Journal of Finance, 32(2), 261-275.

Modigliani, F. y Miller, M. H. (1958). The Cost of Capital, Corporation Finance and the Theory of Investment. The American Economic Review, 48(3), 261-297.

Modigliani, F. y Miller, M. H. (1963), "Corporate income taxes and the cost of capital: A correction", American Economic Review 53, 433-443.

Myers, S. C. (1984). The Capital Structure Puzzle. The Journal of Finance, 39(3), 574-592.

Myers, S. C. (2001). Capital Structure. The Journal of Economic Perspectives, 15(2), 81-102.

Ocampo, A. (2009). Impactos de la Crisis Financiera Mundial sobre Latinoamérica. Revista Cepal, (97).

Puntaier, Elmar. Capital Structure and Profitability: S\&P 500 Enterprises in the Light of the 2008 Financial Crisis, Diplomica Verlag, 2010.

Rivera, R. P., y Campuzano, H. A. (2009). Mercados y crisis financiera global (1996-2008) (Determinantes de la inversión en México).

Rodríguez Puente, Eduardo (2003). "Modelos y prácticas en las decisiones de estructura de capital", $I M E F$, Monterrey.

Standard \& Poors (2021). Income statement, Balance Sheet. 01/01/2004 to 31/12/2019. Extraído el 6 de enero de 2021 de Standard \& Poors database. 
Shyam-Sunder, L. y C. Myers, S. (1999). Testing static tradeoff against pecking order models of capital structure. Journal of Financial Economics, 51(2), 219-244.

Skopljak, V., y Luo, R. H. (2012). Capital structure and firm performance in the financial sector: Evidence from Australia. Asian Journal of Finance \& Accounting, 4(1), 278-298.

Stiglitz, J.E., (1969), "A re-examination of the Modigliani-miller theorem", America Economic Review 59, 784-793.

Zeitun, R., Temimi, A., Mimouni, K. (2017). Do financial crises alter the dynamics of corporate capital structure? Evidence from GCC countries. The Quarterly Review of Economics and Finance, 63, 21-33. 